\title{
Numerical Simulation on Structure Optimization of Liquid-Gas Cylindrical Cyclone Separator
}

\author{
Peng Chang,, Tian Hu, ${ }^{1}$ Li Wang, ${ }^{2}$ Sen Chang, ${ }^{2}$ Tianjing Wang, ${ }^{1}$ and Yueshe Wang ${ }^{1}$ \\ ${ }^{1}$ State Key Laboratory of Multiphase Flow in Power Engineering, Xian Jiaotong University, Xian, China \\ ${ }^{2}$ No. 5 Natural Gas Production Plant, PetroChina Changqing Oilfield Company, Inner Mongolia, China \\ Correspondence should be addressed to Yueshe Wang; wangys@mail.xjtu.edu.cn
}

Received 1 July 2016; Revised 16 August 2016; Accepted 22 August 2016

Academic Editor: Evangelos Tsotsas

Copyright (c) 2016 Peng Chang et al. This is an open access article distributed under the Creative Commons Attribution License, which permits unrestricted use, distribution, and reproduction in any medium, provided the original work is properly cited.

\begin{abstract}
With the further development of oilfield, liquid-gas separation has become an essential problem. Cylindrical cyclone separators are popular in the industrial process due to the advantage that they are simple, compact, and inexpensive to manufacture. In this paper, a three-dimensional turbulence model including Reynolds stress model was established to describe the mixture flow field in the separator. Through the numerical simulation, the separation efficiency was investigated under different parameter cases such as separator length, gas phase outlet diameter, and inlet shape. It can be indicated from the simulation results that the separation efficiency decreases with the increase of the separator length, and the separation efficiency increases firstly and then decreases with the increase of the gas phase outlet diameter as well as the liquid phase outlet. Furthermore, the rectangular inlet is more suitable than the circular inlet with the separation efficiency changing from $66.45 \%$ to $79.04 \%$. In the end, the optimal geometrical structure was presented with separation efficiency of $86.15 \%$.
\end{abstract}

\section{Introduction}

For the past several decades, conventional gravity based separators, such as settling tanks, have been extensively used in industries. Therefore, they are bulky and expensive and show slow separation. Due to these defects, in recent years, the industry has shown keen interest in the development and application of alternatives to the conventional separators. One of such alternatives is the cylindrical cyclone separator (CCS). Unlike the slow settling of particles within a settling tank, a cyclone separator shows fast separation and utilizes less space. It is simply a cylinder installed with a downward inclined tangential inlet and two outlets provided at the top and the bottom, respectively. It operates under the action of centrifugal forces, without any internal moving devices. Fluid mixture enters in the cyclone and makes a swirl motion; because of the centrifugal force, the dense phase of the mixture gains a relative motion in the radial direction and is separated from the mixture. The CCS utilizes the energy obtained from the fluid pressure gradient to create rotational fluid motion instead of using electricity and other types of energy, which leads to relative economy in power usage and flexibility.

The CCS has a variety of potential applications, varying from partial separation to complete separation. It is widely used in industrial processes for separation of dust from gas streams or for product recovery. Potential applications include control of gas/liquid ratio of multiphase flow, portable well test metering, and preseparation upstream of slug catchers [1]. Nowadays, the CCS plays a key role in multiphase flow metering loop. In this area, gas and liquid phases are separated in the CCS. Each of the separated phases is metered by a single-phase flow meter installed in the outlets of the CCS, respectively. After metering, the gas and liquid phases are recombined downstream of the meters to recover twophase flow. Despite its simplicity, the disadvantage of this separator is that the pressure drop and mixture pressure of inlet are high compared with traditional settling chambers.

In order to improve the separating process, primarily, we should understand the fluid dynamics of this separator, such as vorticity and annular zones of reverse flow. Reydon and Gauvin [2] studied the behavior of confined 
vortex flow in conical cyclones. Kouba et al. [3] presented experimental results for air-water system and the effect of inlet inclination angle, operating pressure, and body and inlet geometry on liquid carry-over phenomenon for gas-liquid cylindrical separators. Davies and Watson [4] carried out a study, indicating size, cost, and performance benefits of a modified cyclone over a conventional separator in offshore applications. Gomez et al. [5] proposed a mechanistic model to predict the hydrodynamic flow behavior in a CCS. Four typical systems were designed for actual industrial application, including multiphase metering loop with both singlephase and multiphase flow meters, preseparation, and fullseparation configurations. Arpandi et al. [6] presented new experimental data and an improved mechanism to predict the hydrodynamic flow behavior in the CCS, including the operational envelope, equilibrium liquid level, vortex shape, velocity, and holdup distributions. Hreiz et al. [7] carried out an experiment to study the effect of inlet nozzle design on the performance of the CCS. Theories of confined vortex flow have so far been unable to predict many features of the flow fields observed due to strongly coupled, nonlinear partial differential equations of mass and momentum conservation involved in the fluid dynamics.

With the development of powerful digital computers, this problem can be solved by computational fluid dynamics (CFD). It subdivides the solution domain into a large number of control volumes and converts the partial differential equations by integration over these control volumes into their algebraic equivalents. Boysan et al. [8] presented one of the first CFD models of CCS, showing that the standard $k-\varepsilon$ turbulence model cannot be used for simulating flows with swirl, because it may result in excessive turbulence viscosities and unrealistic tangential velocities. Hoekstra et al. [9] suggested that Reynolds stress model (RSM) can solve the anisotropic turbulence problem, which has been widely used for cyclones and the corresponding numerical simulation in recent studies. Wang et al. [10] presented a numerical study of the gas-powder-liquid flow in a hydrocyclone with the Reynolds stress model, which was verified by the measured results. In recent years, the so-called combined approach of discrete element method (DEM) and CFD (CFD-DEM) has been developed and accounts for both particle-particle and particle-fluid interactions. The CFD-DEM approach has proven to be effective in modeling various particle-fluid flow systems. Chu et al. [11] presented a mathematical model to describe the flow characteristics in dense medium cyclones by means of combining DEM with CFD. The DEM is used to model the motion of discrete particles and the CFD is used to model the motion of discrete particles.

CFD provides an economical means of understanding the complex fluid dynamics and how they are affected by changes in both design and operating conditions. Various CCS have been presented in previous literatures. Crowe and Pratt [12] developed a two-dimensional (2D) model to predict the increase of the overall collection efficiency with solid loading ratio. Movafaghian et al. [13] studied experimentally and theoretically the hydrodynamic flow behavior in CCS. The parameters of this investigation include three different inlet geometries, four different liquid viscosities, three system pressures, and the effect of surfactant. Erdal et al. [14] presented a 2D model of single-phase and two-phase flow in several CCS configurations. Results are also compared with experimental data including tangential velocity profiles and tangential velocity decay, showing good agreement. In fact, if the inlet is ignored, CCS can be considered as symmetric and CFD models can be simplified to a two-dimensional case. Although this method can greatly reduce computational time, it cannot be used to assess changes in inlet design or offset vortex finders. With the development of computer, recent CFD models mostly use full three-dimensional shape to explore flow characteristics in CCS.

Minier and Simonin [15] used a three-dimensional model with Eulerian-Lagrangian approach on a three-dimensional numerical grid applying a modified $k-\varepsilon$ turbulence model. A comparison of the predicted flow field with experimental data was not included in this publication. Furthermore, variations of the coefficients of restitution in the particle-wall model from elastic to completely inelastic bouncing behavior have shown only minor influence on the predicted efficiency grade. Derksen [16] presented a prediction of a flow in a highefficiency cyclone at $\mathrm{Re}=280000$ with numerical investigation. He used calculations using a large eddy simulation (LES) based on the Smagorinsky model, which agree well with experimental data. de Souza and Neto [17] have used subgrid scale Smagorinsky model to predict the behavior of a water-fed hydrocyclone. The numerical results presented the main features of the flow rules and agreed reasonably well with experimental data. The authors suggested that LES represents an interesting alternative to classical turbulence models when applied to numerical solution of fluid flows within hydrocyclones.

According to the above literatures, a large number of researchers were devoted to numerically exploring the flow characteristic and design of CCS. However, up to now, few researchers present a study including all structure factors of CCS, such as shape of inlet and outlet, height, and diameter. In this study, different efficiency caused by different factors is investigated, giving a basis of CCS designing.

\section{Mathematical Model of Liquid-Gas Cylindrical Cyclone Separator}

A classical liquid-gas cylindrical cyclone separator illustrated in Figure 1 is studied for the present computation. The gas and liquid mixtures flow through the inclined inlet section and then, due to the centrifugal force, a swirl causing the gas and liquid to separate is formed. The liquid moves toward the wall and downward, whilst the gas flows toward the center and exits from the upper gas phase outlet.

For the convenience of computation, some assumptions are proposed as follows: (1) the flow state is steady in the separator; (2) the dispersed phase (gas phase) is uniformly distributed in the continuous fluid (liquid phase); (3) the gas and liquid phases are incompressible and the properties of the mixture such as viscosity are constant; and (4) the flow in the cyclone separator is regarded as isothermal; namely, there is no heat transfer between the phases. The conservation equations of the flow phases involved can be written in a 


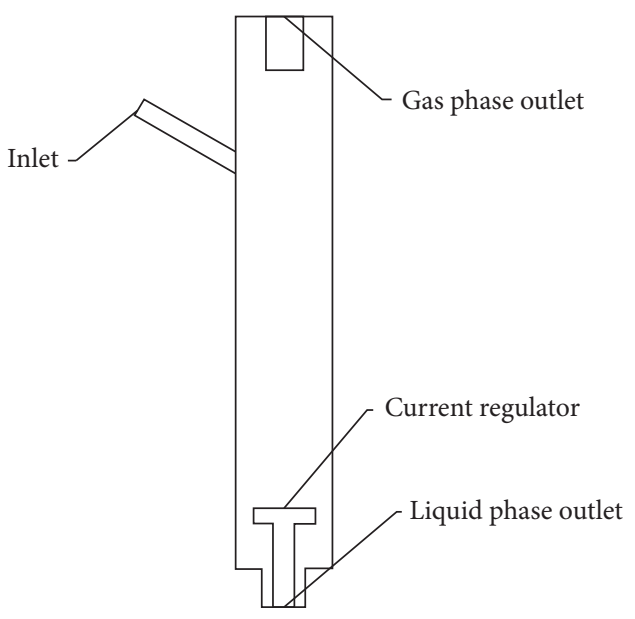

Figure 1: The structure diagram of a classical cylindrical cyclone separator.

generalized form based on the Reynolds-averaging model. The equations are presented as follows:

$$
\begin{aligned}
& \nabla \cdot(\rho \vec{u})=0, \\
& \nabla \cdot(\rho \vec{u} \vec{u})=-\nabla p+\nabla \cdot(\mu \nabla \vec{u})-\nabla \cdot\left(\rho \overline{\vec{u}^{\prime} \vec{u}^{\prime}}\right),
\end{aligned}
$$

in which $\vec{u}$ is the velocity vector, $\mu$ is the viscosity, and $\rho \vec{u}^{\prime} \vec{u}^{\prime}$ is the Reynolds stress tensor.

2.1. Turbulence Model. Numerical methods have been developed to solve (1). At present, standard $k-\varepsilon$ model, RNG $k-\varepsilon$ model, algebraic stress model, and Reynolds stress model are adopted to numerically simulate the turbulence flow of cyclone separators. In the present paper, the flow in the cyclone separator is strongly swirling flow, which has the anisotropic feature. In order to obtain the values of the Reynolds stress terms, the Reynolds stress model was carried out for its adaptation with the strongly anisotropic flow. The Reynolds stress model enables depicting the complex turbulence flow. The Reynolds stress equation is presented as follows:

$$
\frac{\partial \rho \overline{u_{i}^{\prime} u_{j}^{\prime}}}{\partial t}+\bar{u}_{k} \frac{\partial \rho \overline{u_{i}^{\prime} u_{j}^{\prime}}}{\partial x_{k}}=P_{i, j}+\phi_{i, j}+D_{i, j}-\varepsilon_{i, j},
$$

in which $P_{i, j}$ is the stress production term which is ignored in this paper, $\phi_{i, j}$ is the pressure-strain correlation, $D_{i, j}$ is the diffusion term, and $\varepsilon_{i, j}$ is the dissipation rate of the turbulent kinetic energy. They are presented, respectively, as follows:

$$
\begin{aligned}
\phi_{i, j} & =-C_{1} \rho \frac{\varepsilon}{k}\left(\overline{u_{i}^{\prime} u_{j}^{\prime}}-\frac{2}{3} k \delta_{i, j}\right)-C_{2}\left(p_{i, j}-\frac{2}{3} p \delta_{i, j}\right), \\
D_{i, j} & =\frac{\partial}{\partial x_{k}}\left(\frac{\mu_{t}}{\sigma_{t}} \frac{\partial \overline{u_{i}^{\prime} u_{j}^{\prime}}}{\partial x_{k}}\right), \\
\varepsilon_{i, j} & =\frac{2}{3} \rho \varepsilon \delta_{i, j} .
\end{aligned}
$$

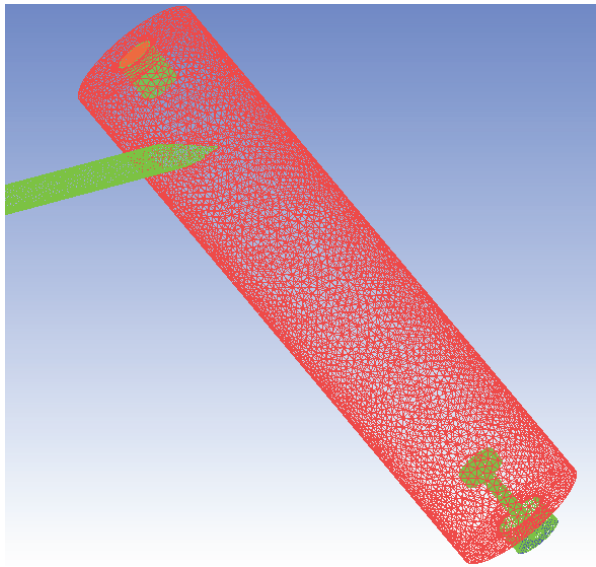

Figure 2: The grid distribution diagram of the cylindrical liquid-gas separator.

Otherwise, the turbulent kinetic energy $k$ and the dissipation rate $\varepsilon$ are given, respectively, by

$$
\begin{aligned}
\frac{D k}{D t}= & \frac{\partial}{\partial x_{l}}\left(c_{k} \frac{k^{2}}{\varepsilon} \frac{\partial k}{\partial x_{l}}+\frac{\mu}{\rho} \frac{\partial k}{\partial x_{l}}\right)-\overline{u_{i}^{\prime} u_{l}^{\prime}} \frac{\partial u_{i}}{\partial x_{l}}-\varepsilon, \\
\frac{D \varepsilon}{D t}= & \frac{\partial}{\partial x_{l}}\left(c_{\varepsilon} \frac{k^{2}}{\varepsilon} \frac{\partial \varepsilon}{\partial x_{l}}+\frac{\mu}{\rho} \frac{\partial \varepsilon}{\partial x_{l}}\right)-c_{\varepsilon 1} \frac{\varepsilon}{k} \overline{u_{i}^{\prime} u_{l}^{\prime}} \frac{\partial u_{i}}{\partial x_{l}} \\
& -c_{\varepsilon 2} \frac{\varepsilon^{2}}{k} .
\end{aligned}
$$

Therefore, the Reynolds stress equations are closed and can be solved.

2.2. Numerical Method and Boundary Condition. Equations were solved numerically through transferring to algebraic equations based on the control volume method. The pressurevelocity coupling algorithm SIMPLE and the QUICK interpolation scheme were used in the numerical simulation. For the consideration of strongly swirling flow in the cyclone separator, the PRESTO! scheme was adopted for pressure discretization. The prototype design of cyclone separator was based on [18]. Figure 2 shows the geometric configuration and mesh distribution of the cyclone separator in this paper, and all the test cases were simulated using unstructured tetrahedral grids generated by a robust method.

Boundary conditions were set as follows: the inlet boundary condition was assumed as velocity inlet condition and the velocities were set for water and methane gas separately. It was assumed that the inlet velocity was uniform. The boundary condition for the wall was regarded as no slip. Finally, the gas phase outlet was regarded as pressure outlet condition as well as the liquid phase outlet, and the pressure was set at atmospheric pressure.

2.3. Turbulence Model Verification. To demonstrate the feasibility of the numerical simulation, a comparative test was carried out. An experiment about the effect of inlet volume flow on the separation efficiency was performed in [18]. In 


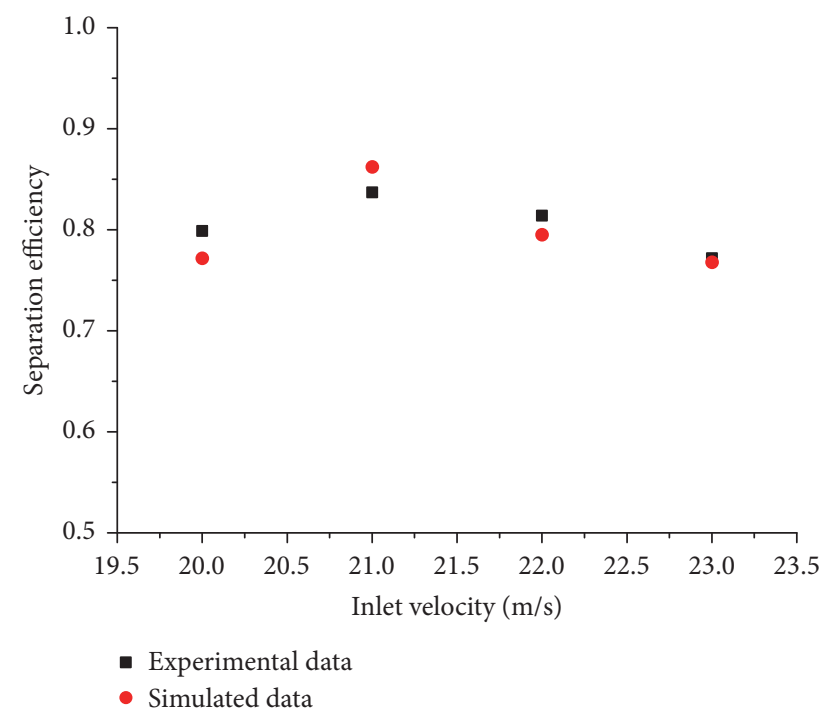

FIGURE 3: The comparison of the simulated data and the experimental data.

the experiment, the mass flow values were set as $11 \mathrm{~m}^{3} / \mathrm{h}$, $11.5 \mathrm{~m}^{3} / \mathrm{h}, 12 \mathrm{~m}^{3} / \mathrm{h}$, and $12.5 \mathrm{~m}^{3} / \mathrm{h}$ separately, corresponding to $20 \mathrm{~m} / \mathrm{s}, 21 \mathrm{~m} / \mathrm{s}, 22 \mathrm{~m} / \mathrm{s}$, and $23 \mathrm{~m} / \mathrm{s}$. The geometric model of the simulation was the same as the separator of the experiment. The inlet is rectangular and the size is $15 \mathrm{~mm} *$ $10 \mathrm{~mm}$, the separator length is $840 \mathrm{~mm}$, the diameter is $100 \mathrm{~mm}$, the gas phase outlet diameter is $26 \mathrm{~mm}$, and the liquid phase diameter is $36 \mathrm{~mm}$. The separation efficiency $\alpha$ was defined as the ratio of the gas phase outlet gas content and the inlet gas content, which can be written as follows:

$$
\alpha=\frac{m_{g, o}}{m_{g, i}} .
$$

The comparison of the simulation and the experiment is shown in Figure 3. It can be indicated that there is a good agreement between the experiment data and the simulation data. Therefore, the simulation method in this paper is applicable and flexible.

\section{Results and Discussion}

In this paper, the effect of some parameters such as separator length, the diameter of the liquid phase outlet and the gas phase outlet, and inlet velocity on the separation efficiency was considered. The values of the parameters were listed in Table 1.

3.1. The Effect of the Separator Length on the Separation Efficiency. The separator length has essential impact on the gas and liquid separation. In this paper, three separators with different lengths are considered. Figure 4 shows the gas volume fraction distribution under three values of length. It can be indicated that, with the increase of the length, the separation performance becomes bad, which can also be deduced from Figure 5 which presents the gas volume
TABLE 1: Different parameters values for simulation test.

\begin{tabular}{lccc}
\hline Case number & 1 & 2 & 3 \\
\hline$L_{s}(\mathrm{~mm})$ & 440 & 840 & 1600 \\
$D_{g}(\mathrm{~mm})$ & 16 & 26 & 36 \\
$D_{l}(\mathrm{~mm})$ & 22 & 36 & 50 \\
\hline Inlet shape & Circular & Rectangular & \\
\hline
\end{tabular}

fraction on the cross section of the gas phase outlet. The reason for the variation of the separation performance may be associated with the tangential velocity and the static pressure distribution. When the length value increases, the liquid-gas mixture stays longer in the cyclone separator, which causes liquid and gas mix again. It can be seen from Figure 6 that the tangential velocity decreases when the length value increases. When the length is $1600 \mathrm{~mm}$, the tangential velocity is almost zero, which demonstrates that the liquid and the gas do not separate. Generally, the separation efficiency is proportional to the tangential velocity. On the other hand, static pressure difference is another important factor for liquidgas separation. The pressure difference supplies centripetal force of the circular motion in the cyclone separator. When the pressure difference is greater, the trend of the liquid-gas separation is more obvious. Figure 7 shows the distribution of the static pressure with different lengths. It indicates that the pressure difference varies in contrast to the variation of the length; therefore, the separation effect becomes worse with the increase of the separator length.

3.2. The Effect of the Gas Phase Outlet Diameter on the Separation Efficiency. The gas phase outlet diameter is another important parameter. When the liquid and gas separate, the gas forms the gas column close to the center of the separator. The gas phase outlet diameter depends on the size of the gas column. The gas volume fraction distribution with three different gas phase outlet diameters is shown in Figure 8. It can be indicated that the volume fraction increases at the beginning and then decreases when the diameter value is larger than a specific value. This can be explained as follows: when the gas phase outlet diameter is very small, the gas column could not flow out completely from the gas phase outlet. When the diameter is very large, larger than the gas column, the gas column will carry the liquid and flow from the gas phase outlet. It can be seen from Figure 9 that although the average tangential velocity is the largest for the case of $r_{g}$ $=13 \mathrm{~mm}$, the difference of the velocity value is not serious.

3.3. The Effect of the Liquid Phase Outlet Diameter on the Separation Efficiency. The liquid phase outlet diameter is also evaluated in this paper. Figure 10 shows the gas volume fraction on the cross section of the gas phase outlet with three different liquid phase outlet diameters. It can be observed that the volume fraction increases at the beginning and then decreases when the diameter value is larger than a specific value. The tangential velocity listed in Figure 11 will give the explanation of the phenomenon. For the case of $r_{l}=18 \mathrm{~mm}$, the average tangential velocity is the largest, which leads to the best separation effect. We can see from Figure 10 that 


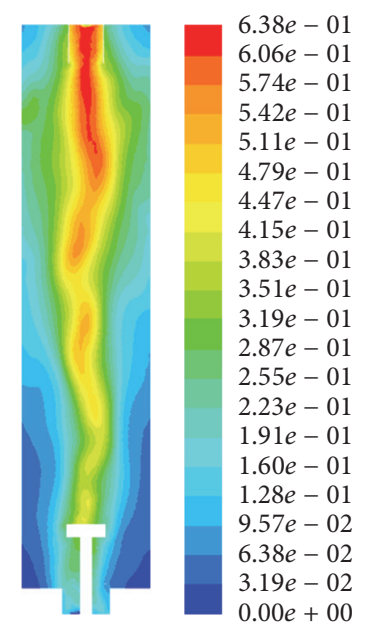

(a)

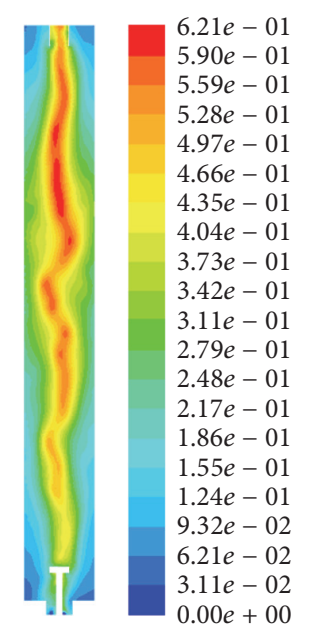

(b)

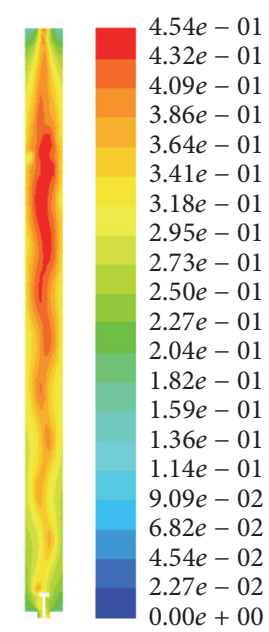

(c)

FIgURE 4: The volume fraction of gas with different lengths of the separator: (a) $L_{s}=440 \mathrm{~mm}$; (b) $L_{s}=840 \mathrm{~mm}$; (c) $L_{s}=1600 \mathrm{~mm}$.

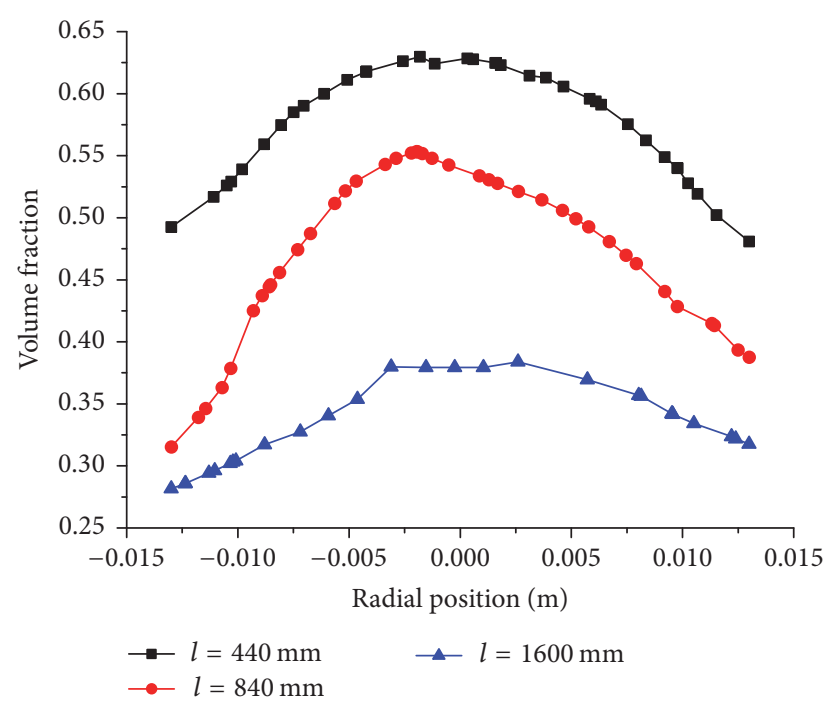

FIGURE 5: The gas volume fraction distribution on the cross section of the gas phase outlet.

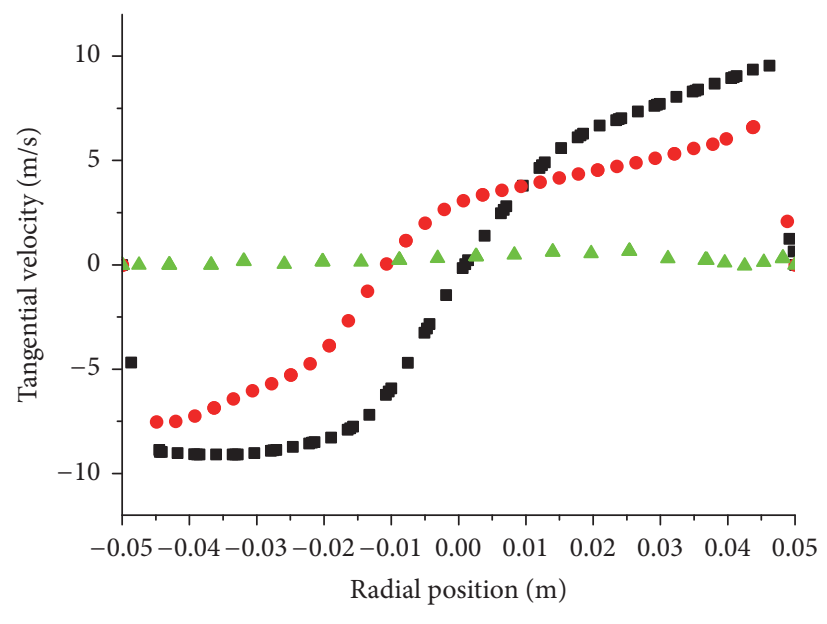

- $l=440 \mathrm{~mm} \quad \Delta l=1600 \mathrm{~mm}$

- $l=840 \mathrm{~mm}$

Figure 6: Tangential velocity distribution on the cross section of the separator middle position. 
TABLE 2: The comparison of separation efficiency with optimized structure.

\begin{tabular}{lcccc}
\hline Case number & 1 & 2 & 3 & 4 \\
\hline Inlet shape and size $(\mathrm{mm})$ & Circular $(D=20)$ & Circular $(D=20)$ & Rectangular $(20 * 20)$ & Rectangular $(20 * 20)$ \\
Length/diameter $(\mathrm{mm} / \mathrm{mm})$ & $840 / 100$ & $440 / 100$ & $840 / 100$ & $440 / 100$ \\
Gas phase outlet diameter $(\mathrm{mm})$ & 13 & 13 & 13 & 13 \\
Liquid phase outlet diameter $(\mathrm{mm})$ & 18 & 18 & 18 & 18 \\
Separation efficiency & $66.45 \%$ & $76.39 \%$ & $79.04 \%$ & $86.15 \%$ \\
\hline
\end{tabular}

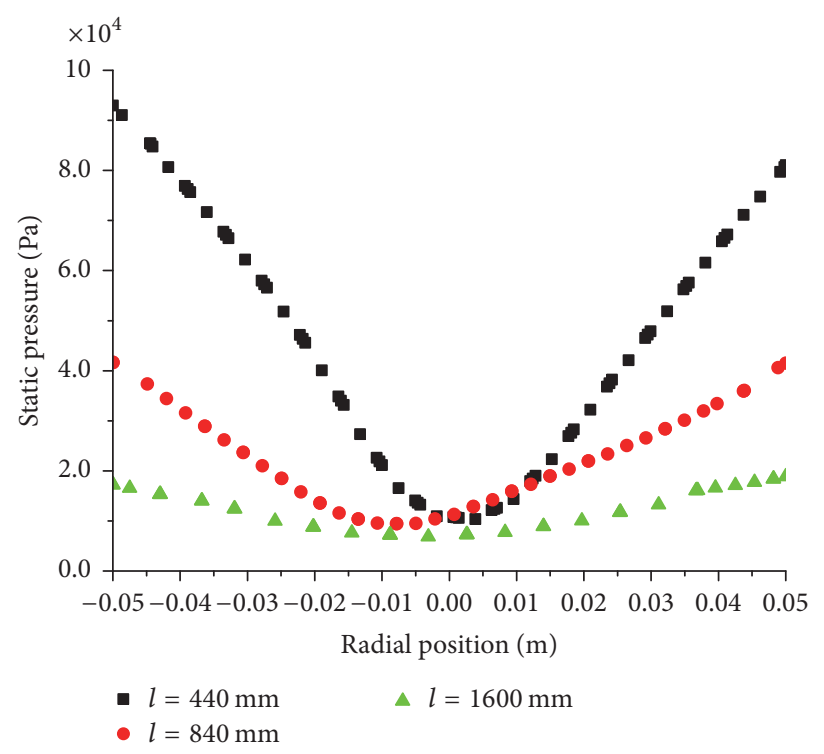

FIGURE 7: Static pressure distribution on the cross section of the separator middle position.

the gas volume fraction increases when the gas is close to the separator center, which demonstrates the existence of the gas column. Figure 11 shows that high tangential velocity occurs in the near-wall region, and it decays rapidly toward the separator center.

\subsection{The Effect of the Inlet Shape on the Separation Efficiency.} The inlet shape plays an important role in the optimal design for the reason that it decides the distribution of the incoming gas and liquid and the initial tangential velocity. In this paper, two inlet shapes are considered, that is, rectangular and circular. Figure 12 presents the gas volume fraction distribution on the cross section of the gas phase outlet for two-inlet shape. The maximum value of the volume fraction for the rectangular inlet is above 0.6 , but for circular inlet it is about 0.54 . In Figure 13, the maximum tangential velocity for the rectangular inlet is $8 \mathrm{~m} / \mathrm{s}$, but for circular inlet it is about $7 \mathrm{~m} / \mathrm{s}$. As a consequence, the separation efficiency is greater for the rectangular inlet than for the circular inlet. For the rectangular inlet, the incoming flow enters the cyclone separator in tangential direction more easily, which causes the tangential velocity to be less lost. Therefore, the rectangular shape is more suitable for cyclone separator.

Through the above simulations and analysis, it can be indicated that the length of the separator should be shorter, the diameter of the gas phase outlet and the liquid phase outlet should be appropriate, and the shape of the inlet should be rectangular. The optimal design parameters are listed in Table 2. The optimal design parameters are case number 4 and the separation efficiency is $86.15 \%$.

\section{Conclusion}

This study carries out the simulation of the flow and liquidgas separation in the cylindrical cyclone separator with different geometric structures and sizes. The gas volume fraction, tangential velocity, and static pressure are presented and analyzed to evaluate the liquid-gas separation. The following conclusions could be drawn from the results obtained in this investigation:

(1) For the case of the separator length, the separation efficiency decreases with the increase of the separator length. But the length should be long enough for full separation of liquid and gas.

(2) The separation efficiency increases firstly and then decreases with the increase of the gas phase outlet diameter, as well as the liquid outlet diameter. 


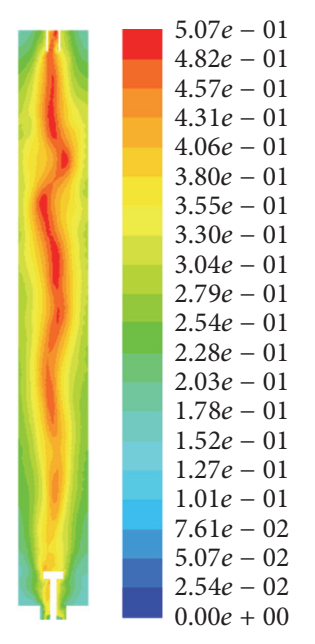

(a)

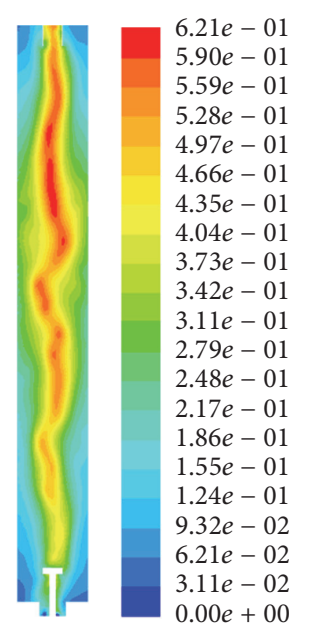

(b)

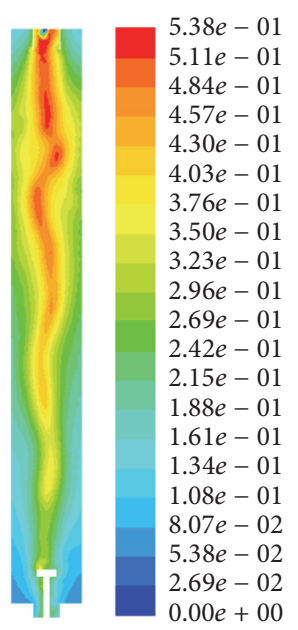

(c)

FIGURE 8: The volume fraction of gas with different gas phase outlet diameters: (a) $r_{g}=8 \mathrm{~mm}$; (b) $r_{g}=13 \mathrm{~mm}$; (c) $r_{g}=18 \mathrm{~mm}$.

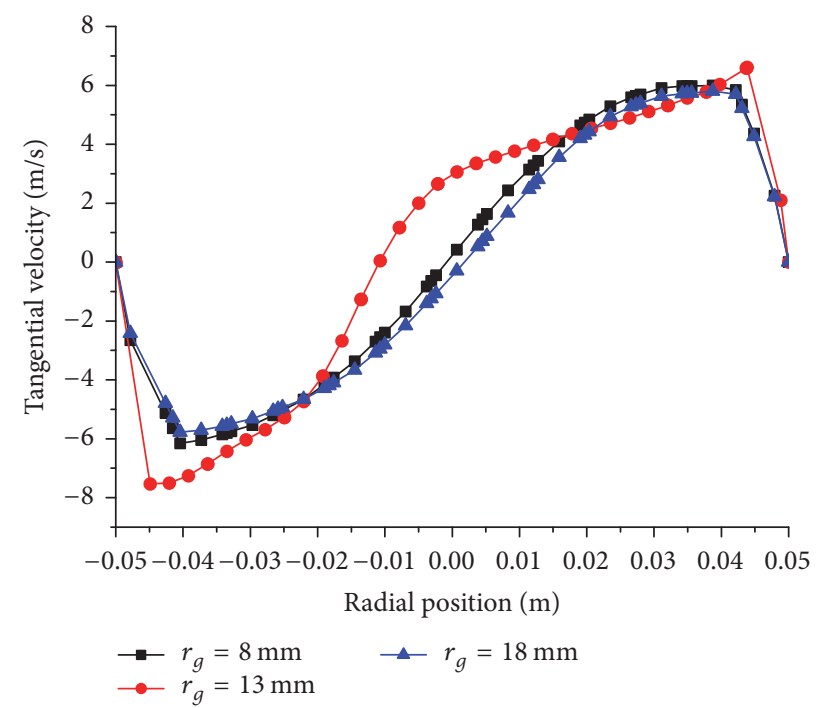

FIGURE 9: Tangential velocity distribution on the cross section of the separator middle position.

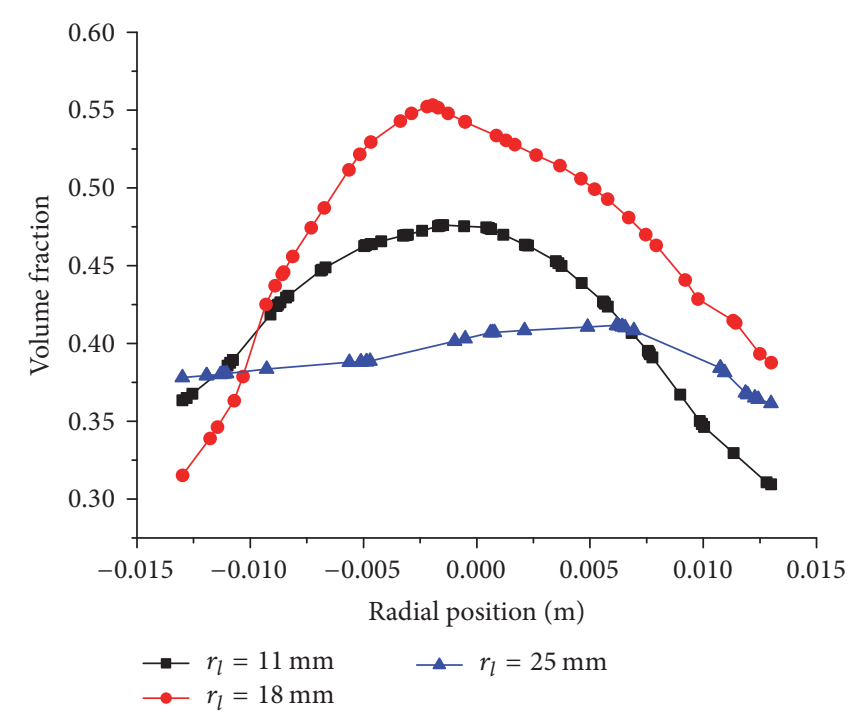

FIGURE 10: The gas volume fraction distribution on the cross section of the gas phase outlet. 


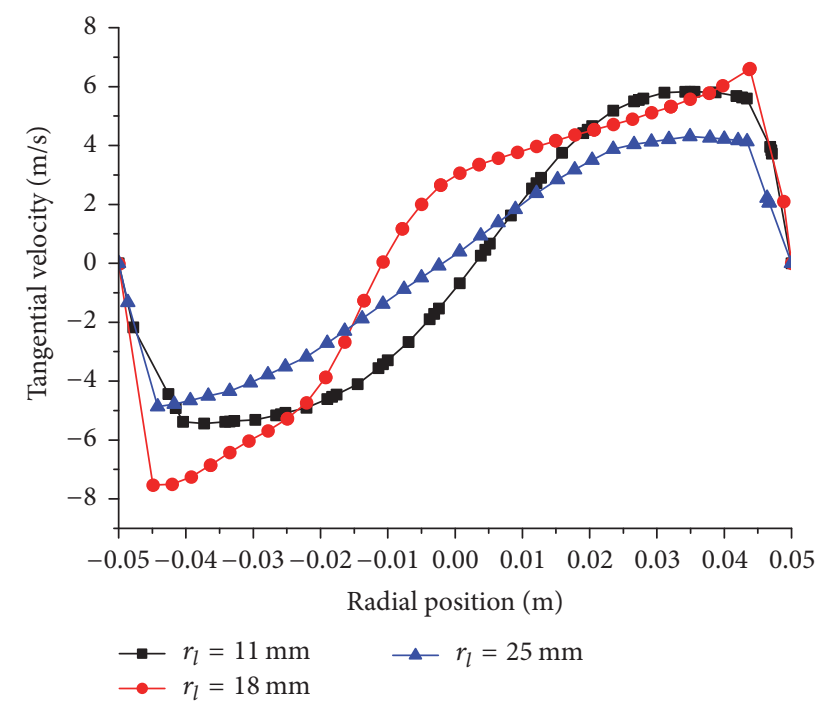

FIGURE 11: Tangential velocity distribution on the cross section of the separator middle position.

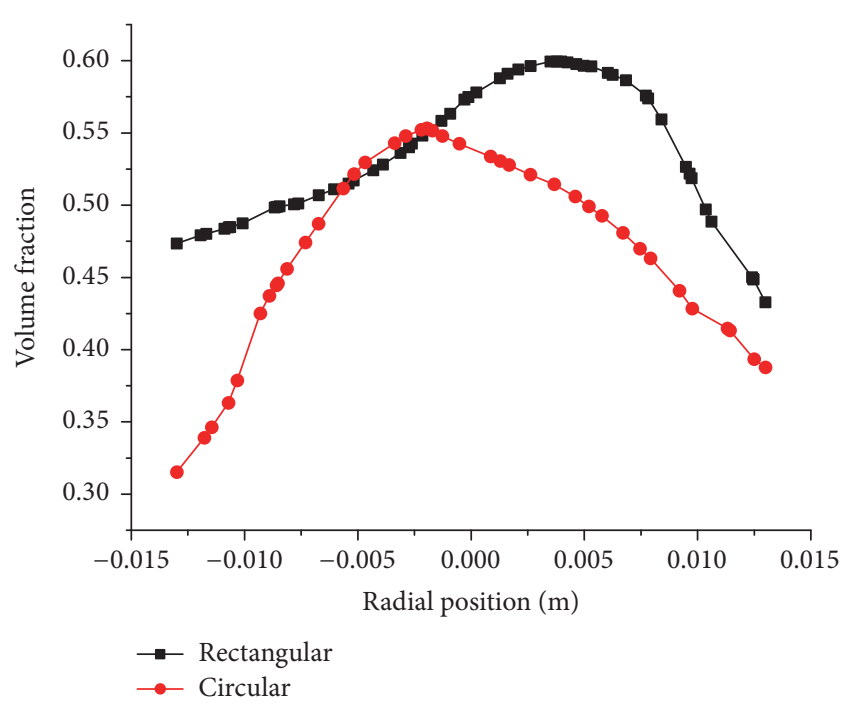

FIGURE 12: The gas volume fraction distribution on the cross section of the gas phase outlet for two-inlet shape.

(3) For the shape of the inlet, the rectangular inlet is more suitable than the circular inlet, and the separation efficiency changes from $66.45 \%$ to $79.04 \%$.

(4) The optimal geometric structure for the cyclone separator is listed as follows: for the rectangular inlet, the length and diameter ratio is $440 / 100$, the gas phase outlet diameter is $13 \mathrm{~mm}$, and the liquid phase outlet diameter is $18 \mathrm{~mm}$. At the same time, the separation efficiency is $86.15 \%$.

\section{Nomenclature}

$D$ : Dynamic viscosity, Pa.s

$k$ : Turbulent kinetic energy

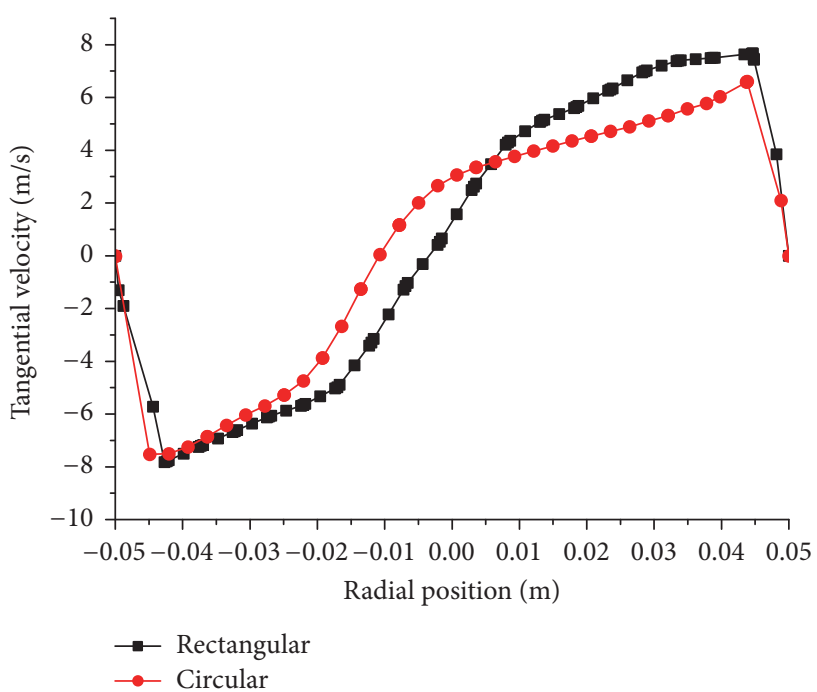

FIGURE 13: Tangential velocity distribution on the cross section of the separator middle position for two-inlet shape.

$m$ : Mass flow rate, $\mathrm{kg} / \mathrm{s}$

$p$ : Static pressure, $\mathrm{Pa}$

$\vec{u}$ : Velocity vector, $\mathrm{m} / \mathrm{s}$

$\mu$ : Dynamic viscosity, Pa.s

$\rho:$ Density, $\mathrm{kg} / \mathrm{m}^{3}$

$\phi$ : Pressure-strain correlation

$\varepsilon$ : Dissipation rate of the

turbulent kinetic energy

$\alpha$ : Separation efficiency.

\section{Competing Interests}

The authors declare that they have no competing interests.

\section{References}

[1] E. G. Arato and N. D. Barnes, "In-line free vortex separator used for gas/liquid separation within a novel two-phase pumping system," in Hydrocyclones, pp. 377-396, Springer, Amsterdam, The Netherlands, 1992.

[2] R. F. Reydon and W. H. Gauvin, "Theoretical and experimental studies of confined vortex flow," The Canadian Journal of Chemical Engineering, vol. 59, no. 1, pp. 14-23, 1981.

[3] G. E. Kouba, O. Shoham, and S. Shirazi, "Design and performance of gas-liquid cylindrical cyclone separators," in Proceedings of the BHR Group 7th International Meeting on Multiphase Flow, pp. 307-327, Cannes, France, June 1995.

[4] E. Davies and P. Watson, "Miniaturized separators for offshore platforms," in Proceedings of the 1st New Technology for Exploration \& Exploitation of Oil and Gas Reserves Symposium, Luxembourg, pp. 75-85, April 1979.

[5] L. E. Gomez, R. S. Mohan, O. Shoham, and G. E. Kouba, "Enhanced mechanistic model and field-application design of gas/liquid cylindrical cyclone separators," SPE Journal, vol. 5, no. 2, pp. 190-198, 2000.

[6] I. Arpandi, A. R. Joshi, O. Shoham, and G. E. Kouba, "Hydrodynamics of two-phase flow in gas-liquid cylindrical cyclone separators," SPE Journal, vol. 1, no. 4, pp. 427-436, 1996. 
[7] R. Hreiz, R. Lainé, J. Wu, C. Lemaitre, C. Gentric, and D. Fünfschilling, "On the effect of the nozzle design on the performances of gas-liquid cylindrical cyclone separators," International Journal of Multiphase Flow, vol. 58, pp. 15-26, 2014.

[8] F. Boysan, W. H. Ayers, and J. Swithenbank, "A fundamental mathematical modelling approach to cyclone design," Transactions of the Institution of Chemical Engineers, vol. 60, no. 4, pp. 222-230, 1982.

[9] A. J. Hoekstra, J. J. Derksen, and H. E. A. Van Den Akker, "An experimental and numerical study of turbulent swirling flow in gas cyclones," Chemical Engineering Science, vol. 54, no. 13-14, pp. 2055-2065, 1999.

[10] B. Wang, K. W. Chu, and A. B. Yu, "Numerical study of particle-fluid flow in a hydrocyclone," Industrial \& Engineering Chemistry Research, vol. 46, no. 13, pp. 4695-4705, 2007.

[11] K. W. Chu, B. Wang, A. B. Yu, and A. Vince, "CFD-DEM modelling of multiphase flow in dense medium cyclones," Powder Technology, vol. 193, no. 3, pp. 235-247, 2009.

[12] C. T. Crowe and D. T. Pratt, "Analysis of the flow field in cyclone separators," Computers \& Fluids, vol. 2, no. 3-4, pp. 249-260, 1974.

[13] S. Movafaghian, J. A. Jaua-Marturet, R. S. Mohan, O. Shoham, and G. E. Kouba, "The effects of geometry, fluid properties and pressure on the hydrodynamics of gas-liquid cylindrical cyclone separators," International Journal of Multiphase Flow, vol. 26, no. 6, pp. 999-1018, 2000.

[14] F. M. Erdal, S. A. Shirazi, O. Shoham, and G. E. Kouba, "CFD simulation of single-phase and two-phase flow in gas-liquid cylindrical cyclone separators," SPE Journal, vol. 2, no. 4, pp. 436-445, 1997.

[15] J. P. Minier and O. Simonin, "A numerical approach of cyclone separators," in Proceedings of the 2nd European Symposium on Separation of Particles from Gases, pp. 121-136, Nuremberg, Germany, March 1992.

[16] J. Derksen, "LES-based separation performance predictions of a stairmand cyclone," in Proceedings of the 10th Workshop on Two-Phase Flow Predictions, Martin-Luther-Universität HalleWittenberg, pp. 217-226, Halle, Germany, 2002.

[17] F. J. de Souza and A. S. Neto, "Large eddy simulations of a hydrocyclone," in Proceedings of the ASME 2002 Joint USEuropean Fluids Engineering Division Conference, pp. 559-564, American Society of Mechanical Engineers, Montreal, Canada, July 2002.

[18] R. Cao, Numerical simulation and structure optimal of columnar gas and liquid separator [M.S. thesis], Daqing Petroleum Institute, 2009. 


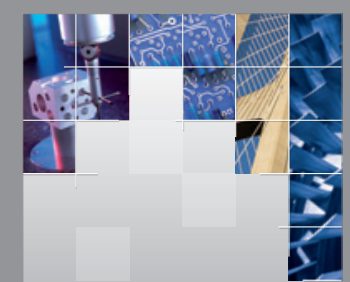

\section{Enfincering}
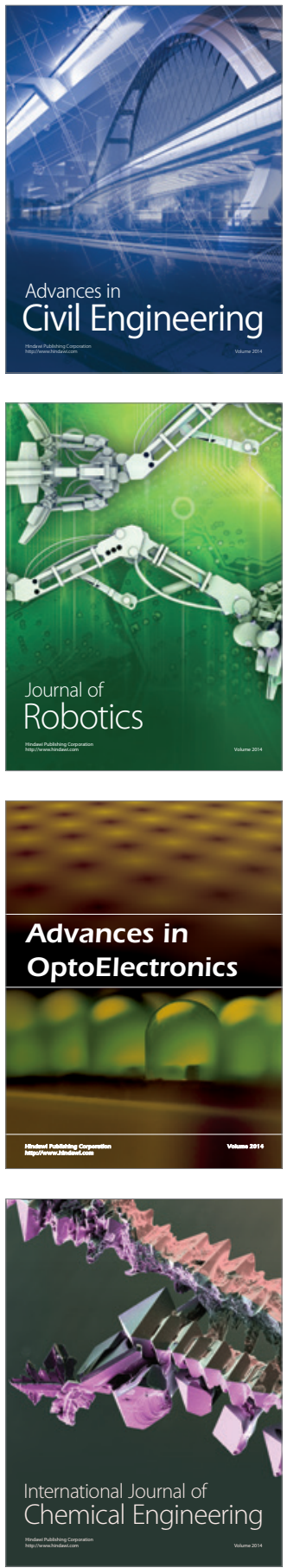

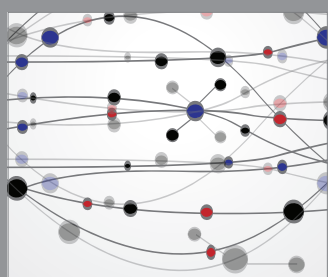

The Scientific World Journal

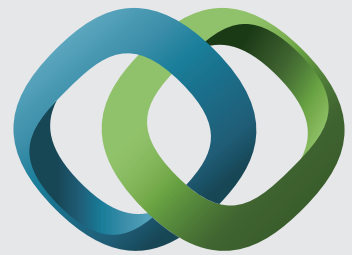

\section{Hindawi}

Submit your manuscripts at

http://www.hindawi.com
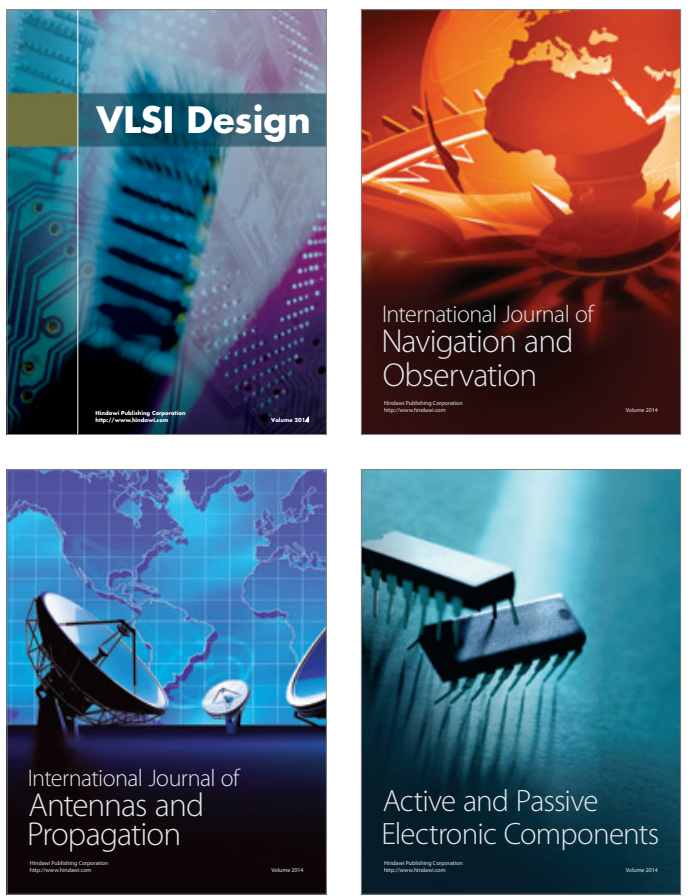
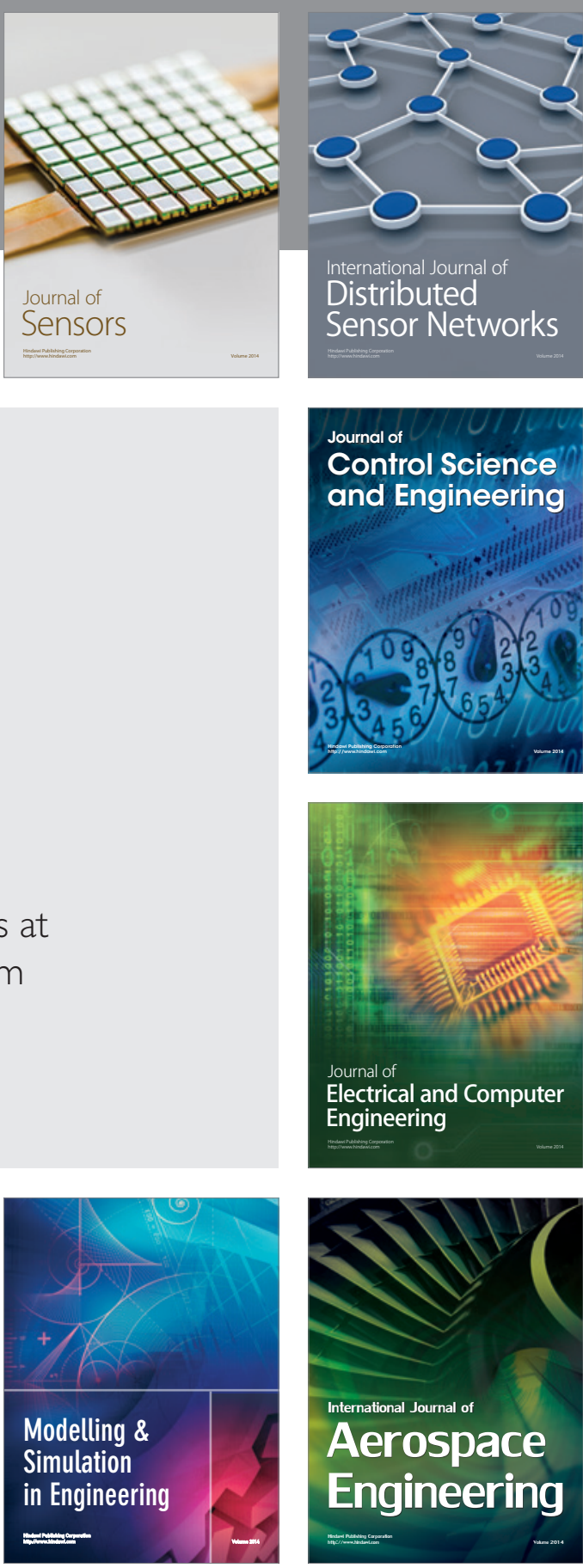

International Journal of

Distributed

Sensor Networks

Journal of

Control Science

and Engineering
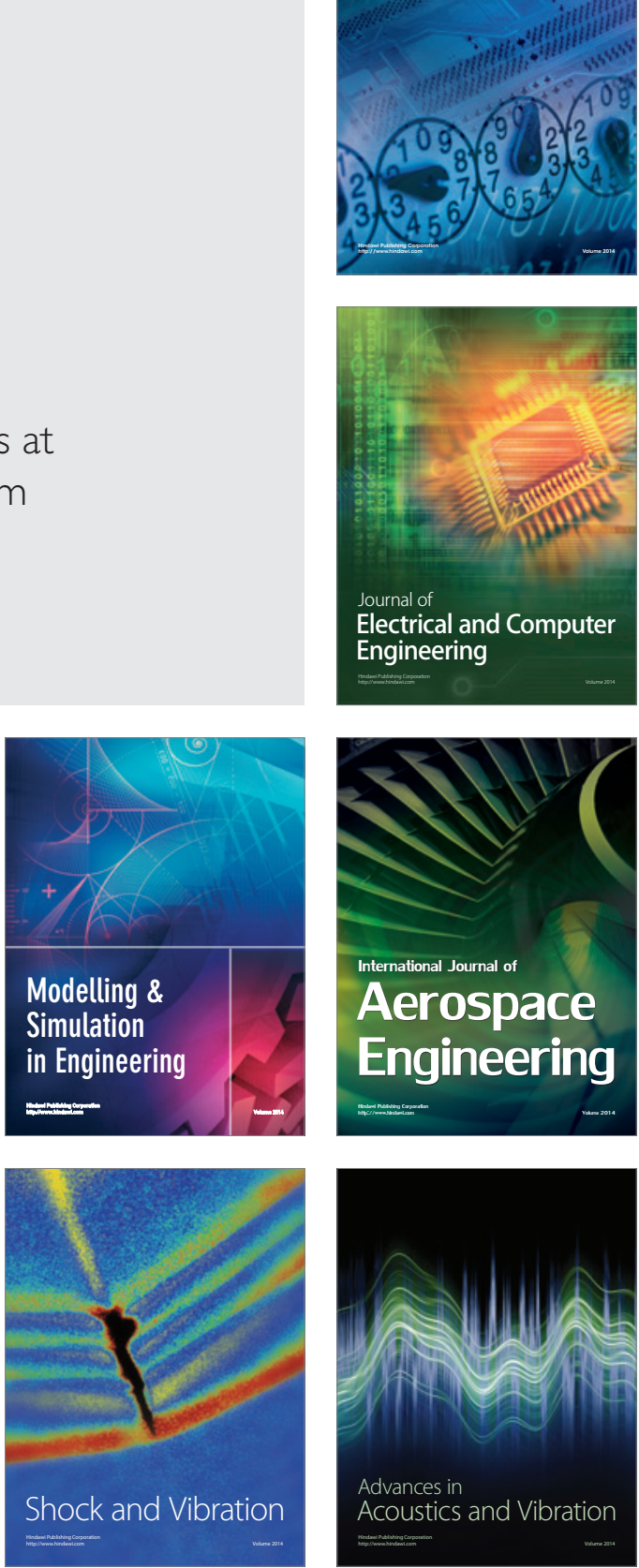